Revista Brasil. Bot., V.32, n.4, p.827-838, out.-dez. 2009

\title{
Comportamento germinativo de espécies epífitas e rupícolas de Bromeliaceae do Parque Estadual do Ibitipoca, Minas Gerais, Brasil ${ }^{1}$
}

\author{
ALBA REGINA PEREIRA ${ }^{2}$, ANTÔNIO CARLOS SILVA DE ANDRADE ${ }^{3,5}$, TÂNIA SAMPAIO \\ PEREIRA $^{3}$, RAFAELA CAMPOSTRINI FORZZA ${ }^{3}$ e ANGELA SAADE RODRIGUES ${ }^{4}$
}

(recebido: 11 de dezembro de 2008; aceito: 30 de setembro de 2009)

\begin{abstract}
Germination behaviour of epiphyte and rupicolous Bromeliaceae species from Ibitipoca State Park, Minas Gerais, Brazil). The germination behaviour of four Bromeliaceae species [Alcantarea imperialis (Carrière) Harms, Pitcairnia flammea Lindl., Vriesea heterostachys (Baker) L. B. Sm. and Vriesea penduliflora L. B. Sm.] from Ibitipoca State Park with contrasting habitat preferences, and representing two different plant growth forms were investigated. The aims of this study were: a) to characterize the seeds, providing information about germination type to describe the post-seminal development morphology; b) to evaluate percentage and rate of germination response to the effect of constant (from $10{ }^{\circ} \mathrm{C}$ to $40{ }^{\circ} \mathrm{C}$ ) and fluctuating $\left(20^{\circ} \mathrm{C}-30{ }^{\circ} \mathrm{C}\right)$ temperatures; c) the ratio of red:far-red light (R:FR) and d) to different water potentials. The results showed that all species have very light seeds, ranging from 0.2 to $1.7 \mathrm{mg}$; the germination is epigeal with cryptocotylar seedlings. All species presented high germination under temperatures between $20^{\circ} \mathrm{C}$ and $30{ }^{\circ} \mathrm{C}$. Under darkness germination was successful only for Vriesea species, but seeds of all species required light for maximum germination. The R:FR value that resulted in 50\% of maximum germination varied among species. Water stressed conditions reduced the percentage and rate of germination in all bromeliad species. A. imperialis and $P$. flammea were less sensitive to low water potentials. These contrasting germination requirements among the species studied were related to their habitat preferences.
\end{abstract}

Key words - red:far-red ratio, rupestrian fields, seedling morphology, temperature, water potential

RESUMO - (Comportamento germinativo de espécies epífitas e rupícolas de Bromeliaceae do Parque Estadual do Ibitipoca, Minas Gerais, Brasil). Estudou-se o comportamento germinativo de quatro espécies de Bromeliaceae [Alcantarea imperialis (Carrière) Harms, Pitcairnia flammea Lindl., Vriesea heterostachys (Baker) L. B. Sm. e Vriesea penduliflora L. B. Sm.], oriundas do Parque Estadual do Ibitipoca - MG, com preferências contrastantes de habitat e representando duas formas de vida diferentes. Os objetivos do presente estudo foram: a) caracterizar as sementes quanto ao tipo de germinação e descrever a morfologia do desenvolvimento pós-seminal; b) avaliar o efeito das temperaturas constantes $\left(10^{\circ} \mathrm{C}\right.$ a $\left.40{ }^{\circ} \mathrm{C}\right)$ e alternada (20-30 $\left.{ }^{\circ} \mathrm{C}\right)$; c) da razão vermelho:vermelho-extremo (V:VE) e d) dos diferentes potenciais de água na porcentagem e na velocidade de germinação das sementes. Os resultados demonstraram que todas as espécies produzem sementes pequenas e muito leves, variando de 0,2 a 1,7 mg; a germinação é epígea com plântulas criptocotiledonares e apresentaram valores ótimos de temperatura entre $20{ }^{\circ} \mathrm{C}$ e $30{ }^{\circ} \mathrm{C}$. Vriesea heterostachys e $\mathrm{V}$. penduliflora foram as únicas que germinaram com sucesso no escuro, mas as sementes das quatro espécies requerem luz para a máxima germinação. Os valores de V:VE que resultaram em 50\% da máxima germinação variaram entre as espécies. As condições de estresse hídrico reduziram a porcentagem e a velocidade de germinação de forma distinta entre as espécies. A. imperialis e P. flammea foram menos sensíveis aos baixos potenciais hídricos. Os requerimentos germinativos contrastantes entre as espécies estudadas estão relacionados à preferência de habitat de cada uma delas.

Palavras-chave - campos rupestres, morfologia de plântulas, potencial hídrico, razão vermelho:vermelho-extremo, temperatura,

\section{Introdução}

Das diferentes fases do ciclo de vida dos vegetais, a germinação de sementes pode determinar a distribuição

1. Parte da tese de doutorado da primeira autora, apresentada ao Programa de Pós-Graduação em Botânica do Jardim Botânico do Rio de Janeiro.

2. Universidade do Estado do Rio de Janeiro, Departamento de Biologia Celular e Genética, Maracanã, Pavilhão Haroldo Lisboa da Cunha, sala 218, 20550-013 Rio de Janeiro, RJ, Brasil.

3. Instituto de Pesquisas Jardim Botânico do Rio de Janeiro, Diretoria de Pesquisa Científica, Rua Pacheco Leão 915, 22460-030 Rio de Janeiro, RJ, Brasil.

4. Pesquisadora Associada, Pontífice Universidade Católica, RJ.

5._Autor para correspondência: candrade@jbrj.gov.br das espécies nos diferentes habitats. O estudo dessa fase é de fundamental importância tanto para a compreensão do estabelecimento de uma comunidade vegetal como para a sobrevivência e regeneração natural das espécies (Fenner \& Thompson 2005). Sob condições naturais, a germinação é uma fase restrita a locais onde são encontradas condições específicas e satisfatórias para o recrutamento de plântulas (Grime 2001, Pearson et al. 2002). Estes microambientes favoráveis e com baixa competição são freqüentemente chamados "sítios seguros" (Harper 1977) ou "nichos de regeneração" (Grubb 1977). O controle sobre a germinação nestes locais é exercido por fatores físicos do ambiente, principalmente temperatura, luz e água, promovendo o 
surgimento de características germinativas diversificadas entre as espécies (Baskin \& Baskin 2001).

De maneira geral, acredita-se que espécies congenéricas e com distribuição geográfica semelhante sejam evolutivamente mais próximas e que tenham atributos de história de vida similares. Desta forma, qualquer diferença nas respostas ecológicas entre estas espécies poderia indicar possíveis adaptações ao habitat ocupado (Van Assche et al. 2002). A comparação entre as exigências de germinação de espécies evolutivamente mais próximas pode indicar como o processo de germinação é adaptado às condições do habitat e como é controlado pelos fatores ambientais.

Bromeliaceae compreende 58 gêneros e, aproximadamente, 3.172 espécies (Luther 2008), sendo que mais de $40 \%$ das espécies dessa família são nativas do Brasil (Leme 1997). O levantamento realizado no Parque Estadual do Ibitipoca (PEIB) indicou a ocorrência de 32 espécies de bromélias que podem ocorrer como rupícolas, terrestres ou epífitas (Monteiro \& Forzza 2008). Esse Parque está situado no Domínio da Floresta Atlântica, no sudeste de Minas Gerais e apresenta um mosaico de comunidades vegetais, composto principalmente por campos rupestres entremeados por floresta estacional semidecidual, floresta ombrófila densa e matas nebulares (Dias et al. 2002). Este local foi especialmente selecionado para este estudo pela diversidade de habitats e de espécies de bromélias com hábitos distintos de crescimento.

A composição e distribuição de comunidades de bromélias epífitas no dossel das florestas estão diretamente relacionadas à habilidade das espécies em explorar gradientes microclimáticos, principalmente de luz e umidade (Benzing 2000). No interior das florestas o gradiente de luz é de extrema importância, modulando a estratificação vertical em espécies epífitas, onde algumas delas desenvolvem-se com alta irradiação e outras sob extremo sombreamento (Graham \& Andrade 2004). A estratificação vertical em bromélias epífitas tem sido relacionada com a tolerância à baixa irradiação solar dessas plantas (Cervantes et al. 2005). Em contrapartida, as espécies rupícolas desenvolvem-se em ambientes com alta irradiação solar, alta variação de temperatura, exposição a ventos fortes e escassez de água e nutrientes (Burke 2002), constituindo-se um ambiente restrito para a maioria das espécies de plantas. Embora as superfícies rochosas apresentem barreiras ecológicas para a germinação de sementes e estabelecimento de plântulas, as famílias Bromeliaceae, Eriocaulaceae, Orchidaceae, Velloziaceae, Cyperaceae e Cactaceae são frequentemente encontradas nesse tipo de habitat (Coan et al. 2002, Ribeiro et al. 2007).

Este artigo teve como objetivos comparar a germinação das espécies quanto à resposta a temperatura, qualidade de luz (razão vermelho:vermelho-extremo) e disponibilidade hídrica, bem como descrever o desenvolvimento pós-seminal de duas Bromeliaceae rupícolas, Alcantarea imperialis (Carrière) Harms e Pitcairnia flammea Lindl., e duas epífitas, Vriesea heterostachys (Baker) L. B. Sm. e Vriesea penduliflora L. B. Sm. Como as quatro espécies apresentam-se muito próximas geograficamente, pergunta-se se o comportamento germinativo poderia explicar a preferência das espécies pelos habitats epifítico ou rupestre.

\section{Material e métodos}

Frutos maduros de A. imperialis, P. flammea, $V$. heterostachys e $V$. penduliflora foram coletados no Parque Estadual do Ibitipoca, MG, situado entre os municípios de Lima Duarte e Santa Rita do Ibitipoca $\left(21^{\circ} 40^{\prime}-21^{\circ} 44^{\prime} \mathrm{S}\right.$ e $\left.43^{\circ} 52^{\prime}-43^{\circ} 55^{\prime} \mathrm{W}\right)$.O relevo é fortemente escarpado com altitudes variando entre 1.200 a 1.784 metros (Menini Neto et al. 2007). O clima da região apresenta chuvas elevadas no verão e inverno frio e seco, sendo classificado como tropical de altitude mesotérmico. Na época mais fria a média de temperatura varia entre $12^{\circ} \mathrm{C}$ a $15^{\circ} \mathrm{C}$ e na época mais quente entre $18{ }^{\circ} \mathrm{C}$ a $22^{\circ} \mathrm{C}$ (Rodela \& Tarifa 2002). A coleta dos frutos foi feita no período de maio de 2005 a dezembro 2006. Informações sobre as quatro espécies estudadas (subfamílias, locais e datas de coleta) e suas principais características (hábito e habitat) são apresentadas na tabela 1. As sementes foram beneficiadas e armazenadas sob temperatura de $10^{\circ} \mathrm{C}$ por 15 dias, no Laboratório de Sementes do Instituto de Pesquisas Jardim Botânico do Rio de Janeiro.

Para a descrição biométrica das sementes (comprimento e largura) foram escolhidas ao acaso 20 sementes de 5 indivíduos diferentes por espécie e tomadas medidas com paquímetro digital (Mitutoyo). O comprimento e largura das sementes foram representados pela média ( \pm desvio padrão) dos dados. O peso de 1.000 sementes foi determinado utilizando-se balança analítica (0,0001 g) segundo as Regras para Análise de Sementes (Brasil 1992).

Nos experimentos de germinação, as sementes foram semeadas em placas de Petri (diâmetro $5 \mathrm{~cm}$ ) contendo duas folhas de papel de filtro umedecidas com $1 \mathrm{~mL}$ de água destilada. A temperatura utilizada nos experimentos de luz, estresse hídrico e para a descrição do desenvolvimento pósseminal das espécies foi a que promoveu os mais altos valores de porcentagem e de velocidade de germinação, verificada no experimento de temperatura. Os parâmetros para avaliação das melhores condições de germinação foram: o índice de velocidade de germinação de Maguire (Santana \& Ranal 
Tabela 1. Informações gerais sobre as quatro espécies coletadas no Parque Estadual de Ibitipoca, Lima Duarte, MG.

Table 1. General information of four collected species from Ibitipoca State Park, Lima Duarte, MG

\begin{tabular}{|c|c|c|c|c|c|}
\hline Subfamília & Espécie & Local de coleta & Data de coleta & Hábito & Habitat \\
\hline Pitcairnioideae & $\begin{array}{l}\text { Alcantarea imperialis } \\
\text { (Carrière) Harms. }\end{array}$ & Campo rupestre & Dezembro & Rupícola & $\begin{array}{l}\text { Afloramentos rochosos } \\
\text { de quartzito, solos rasos. } \\
\text { Alta intensidade de luz, } \\
\text { variação térmica alta, baixa } \\
\text { disponibilidade de nutrientes } \\
\text { e água no solo, ventos fortes. }\end{array}$ \\
\hline Tillandsioideae & $\begin{array}{l}\text { Vriesea heterostachys } \\
\text { (Baker) L. B. Sm. }\end{array}$ & $\begin{array}{l}\text { Floresta atlântica } \\
\text { (sub-bosque de } \\
\text { floresta ombrófila) }\end{array}$ & Maio & $\begin{array}{l}\text { Epífita ou } \\
\text { terrestre }\end{array}$ & $\begin{array}{l}\text { Desenvolvem-se em árvores } \\
\text { e beneficiam-se da umidade } \\
\text { e minerais disponíveis nos } \\
\text { troncos. Podem ocorrer no } \\
\text { solo da mata como terrestre. }\end{array}$ \\
\hline Tillandsioideae & $\begin{array}{l}\text { Vriesea penduliflora } \\
\text { L. B. Sm. }\end{array}$ & $\begin{array}{l}\text { Floresta atlântica } \\
\text { (dossel e bordas } \\
\text { da mata nebular) }\end{array}$ & Maio & $\begin{array}{l}\text { Epífita ou } \\
\text { terrestre }\end{array}$ & $\begin{array}{l}\text { Baixa intensidade de luz, alta } \\
\text { disponibilidade de nutrientes e } \\
\text { água. Variação térmica baixa. }\end{array}$ \\
\hline
\end{tabular}

2004) e a porcentagem de sementes germinadas a partir da protrusão da raiz primária (1 mm), com geotropismo positivo, para $P$. flammea, e protrusão do cotilédone para A. imperialis, $V$. heterostachys e $V$. penduliflora. Em todos os experimentos, a germinação foi verificada diariamente, exceto para o experimento de qualidade de luz, onde foi avaliada apenas a germinação final após 20 dias. Os tratamentos foram esquematizados em delineamento inteiramente casualizado, com quatro repetições de 25-50 sementes cada, dependendo da espécie estudada.

No estudo da morfologia da semente e do desenvolvimento pós-seminal, as ilustrações foram realizadas com o auxílio de estereomicroscópio óptico (Zeiss), equipado com câmara clara. A terminologia adotada na descrição das plântulas baseou-se em Pereira (1988) e em Tillich (2007). Os critérios estabelecidos para caracterizar a plântula normal em cada espécie foram: desenvolvimento radicular sadio, expansão total da primeira folha e aparecimento da segunda folha; e para planta jovem o aparecimento da terceira folha.

A determinação da temperatura ideal para a germinação foi avaliada nas temperaturas constantes de $10^{\circ} \mathrm{C}, 15^{\circ} \mathrm{C}$, $20^{\circ} \mathrm{C}, 25^{\circ} \mathrm{C}, 30^{\circ} \mathrm{C}, 35^{\circ} \mathrm{C}$ e $40^{\circ} \mathrm{C}$ e alternada de $20^{\circ} \mathrm{C}-30^{\circ} \mathrm{C}$ (8/16 h, respectivamente). Foi usado o fotoperíodo de 8 h, com quatro lâmpadas brancas fluorescentes de $20 \mathrm{~W}$ e densidade de fluxo de fótons de $90 \mu \mathrm{mol} \mathrm{m}{ }^{-2} \mathrm{~s}^{-1}(\mathrm{~V}: \mathrm{VE} \cong 6,5)$.

As respostas aos tratamentos de luz foram avaliadas sob temperatura constante ótima, obtida no experimento anterior, inicialmente comparando a condição de luz (fotoperíodo $8 \mathrm{~h}$ ) e de escuro. Posteriormente, testou-se a razão entre os comprimentos de onda correspondentes ao vermelho $(675-685 \mathrm{~nm})$ e vermelho-extremo (725-735 nm) (V:VE), simulando diferentes níveis de sombreamento encontrados sob condições naturais. Filmes verdes de poliéster recobriram as placas de Petri e produziram os seguintes valores de V:VE, com os respectivos valores de densidade de fluxo de fótons entre parênteses ( $\left.\mu \mathrm{mol} \mathrm{m}{ }^{-2} \mathrm{~s}^{-1}\right)$ : 1,20 (48,41); 0,43 (20,77); 0,27 (18,12); 0,13 (3,88); 0,06 (13,46); 0,03 (4,16); e escuro. $\mathrm{O}$ mais alto valor de $\mathrm{V}$ :VE utilizado neste estudo é semelhante à da luz solar (1,19 V:VE; Pons 2000). As placas de Petri foram iluminadas com duas lâmpadas fluorescentes de $22 \mathrm{~W}$ e seis lâmpadas incandescentes de 15 W, sob fotoperíodo de 8 horas. A condição de escuro foi obtida com a colocação das placas de Petri dentro de caixas plásticas pretas do tipo gerbox. A densidade de fluxo de fótons e diferentes valores de V:VE foram medidas com auxílio dos sensores SKR 110 e SKP 215, respectivamente, acoplados ao SpectroSense (Skye Instruments Inc.).

Para simular a redução de disponibilidade hídrica do substrato foram utilizados os potenciais hídricos $\left(\Psi_{\mathrm{w}}\right)$ de 0,0 (água destilada); -0,25 MPa; -0,5 MPa; -0,75 MPa; -1,0 MPa e -1,25 MPa. As concentrações de PEG 6.000 usadas para cada $\Psi_{\text {w }}$ foram calculadas de acordo com Michel \& Kaufmann (1973).

$\mathrm{Na}$ análise dos dados de germinabilidade, quando as pressuposições básicas de normalidade e homogeneidade das variâncias não foram atendidas, seus valores foram transformados para arco seno da raiz quadrada de x/100 (Zar 1999). A normalidade foi avaliada pelo teste de KolmogorovSmirnov e a homogeneidade das variâncias pelo teste de Bartlett (Santana \& Ranal 2004). A diferença entre as médias foi indicada pelo teste $t$ (comparação germinação luz $x$ escuro) ou pela análise de variância, com comparação entre médias 
pelo teste de Tukey. A relação entre a porcentagem final de germinação e os valores de V:VE foi analisada através de regressão logística sigmoidal, de acordo com Pearson et al. (2003): $\mathrm{y}=a /\left\{1+\exp -\left[\left(\mathrm{x}-\mathrm{x}_{0}\right) b\right]\right\}$, onde $a$ é o coeficiente que descreve a máxima porcentagem de germinação, $b$ é o coeficiente de inclinação da resposta de germinação, calculado pelas estimativas de V:VE a 75\% e 25\% da máxima germinação, e xo (ou V:VE $50 \%$ Gmáx $)$ é o coeficiente que estima o valor de V:VE na qual 50\% da máxima germinação ocorre, sendo utilizado na comparação entre espécies. As análises estatísticas foram feitas com auxílio do programa Statistica 6.0 (Statsoft, Inc 2001).

\section{Resultados}

Morfologia de sementes e do desenvolvimento pósseminal - A morfologia da semente e do desenvolvimento pós-seminal de $V$. heterostachys e $V$. penduliflora (figura 1 ), foram muito similares. Ambas as espécies apresentam sementes pequenas, filiformes, com aproximadamente 6,4 $\mathrm{mm}$ de comprimento e 1,1 mm de largura (figura $1 \mathrm{~A}$ e F), com apêndices plumosos esbranquiçados, presentes apenas em uma das extremidades da semente, com tegumento castanho, e peso de 1.000 sementes de aproximadamente $0,47 \mathrm{~g}$. Do ponto de vista morfológico, a germinação iniciou-se aos 4-5 dias de embebição, com a emergência da base do cotilédone, e posteriormente desenvolveu-se a raiz primária (figura 1B e G). Durante o desenvolvimento pós-seminal o haustório é visível por transparência no interior dos restos seminais (figura 1B). As plântulas aos 10-12 dias apresentam a raiz primária recoberta pela coifa, bainha cotiledonar túrgida e hipocótilo pouco desenvolvido (figura 1B). A plântula normal tem bainha cotiledonar transpassada profundofendida (figura 1C e H); eófilo e raiz primária truncadas e recobertas pela coifa (figura 1D e I). As plantas jovens apresentam as folhas subseqüentes imbricadas em roseta (figura 1E e J) e raiz primária truncada recoberta ou não pela coifa aos 20-21 dias.

Observou-se que a morfologia da semente e do desenvolvimento pós-seminal de A. imperialis assemelha-se com as espécies de Vriesea. No entanto, as sementes filiformes de $A$. imperialis são maiores, com aproximadamente $8,7 \mathrm{~mm}$ de comprimento e 1,0 mm de largura, com apêndices plumosos esbranquiçados, presentes nas duas extremidades da semente (figura $1 \mathrm{~K})$, com tegumento marrom-escuro, brilhoso, e peso de 1.000 sementes de 1,656 g. A germinação iniciou-se aos sete dias de embebição, com a emergência da base do cotilédone (figura 1L), e posteriormente desenvolveu-se raiz primária curta ou ausente (figura $1 \mathrm{M}$ e N). As plântulas com 15 dias apresentam a bainha cotiledonar túrgida e raiz primária recoberta pela coifa (figura $1 \mathrm{~N}$ ). A plântula normal tem bainha cotiledonar ligulada profundo-fendida; eófilo e raiz primária em desenvolvimento, hipocótilo pouco desenvolvido ou ausente, ou raiz primária truncada e recoberta pela coifa (figura $1 \mathrm{~N}$ ). As plantas jovens apresentam folhas subseqüentes imbricadas em roseta podendo apresentar raiz primária desenvolvida, freqüentemente truncada com pêlos junto ao colo, recoberta ou não pela coifa aos 30 dias (figura 10).

Em contrapartida, a espécie pertencente à subfamília Pitcairnioideae, $P$. flammea, apresenta diferenças marcantes quando comparadas com as três espécies de Tillandsioideae. Pitcairnia flammea apresenta sementes menores, com aproximadamente $1,8 \mathrm{~mm}$ de comprimento e 0,9 mm de largura, elípticas, bicaudadas, com alas membranáceas (figura 1P), tegumento marromavermelhado, e com peso de 1.000 sementes 0,162 g. Do ponto de vista morfológico, a germinação iniciou-se aos 12 dias de embebição, pelo rompimento dos tegumentos e protrusão da raiz primária recoberta pela coifa (figura 1Q). Durante o desenvolvimento pós-seminal observa-se geotropismo positivo da raiz primária e o cotilédone se eleva da superfície do substrato por alongamento do hipocótilo (figura 1Q). As plântulas apresentam hipocótilo conspícuo que se alonga e eleva do substrato a porção emergente foliácea do cotilédone e raiz primária bem desenvolvida, com colo bem delimitado por um anel de pêlos (figura 1R). A plântula normal apresenta bainha cotiledonar foliácea e exibe o eófilo lanceolado aos 15 dias; o hipocótilo é branco, translúcido, cilíndrico, com pêlos glandulares esparsos (figura 1S). A planta jovem apresenta raiz primária desenvolvida, colo bem delimitado, hipocótilo cilíndrico, creme-esverdeado, com raízes adventícias e bainha cotiledonar foliácea lanceolada com base profundamente fendida, com folhas subseqüentes imbricadas em roseta aos 25 dias. Escamas foliares esparsas recobrem a lâmina foliar e o hipocótilo (figura 1T).

A morfologia do desenvolvimento pós-seminal em Bromeliaceae é determinantemente diagnóstica entre as subfamílias, apresentando diferenças nas suas estruturas essenciais, porém, alguns caracteres não diferenciam os gêneros. Entre as subfamílias estudadas, as plântulas de Vriesea e Alcantarea (Tillandsioideae), embora tenham dimensões diferentes, apresentam as mesmas características morfológicas em suas estruturas essenciais. No entanto, as espécies de Tillandsioideae diferem de Pitcairnioideae, e a estrutura mais diagnóstica é o hipocótilo, conspícuo em Pitcairnia e ausente ou muito reduzido nas Tillandsioideae. 


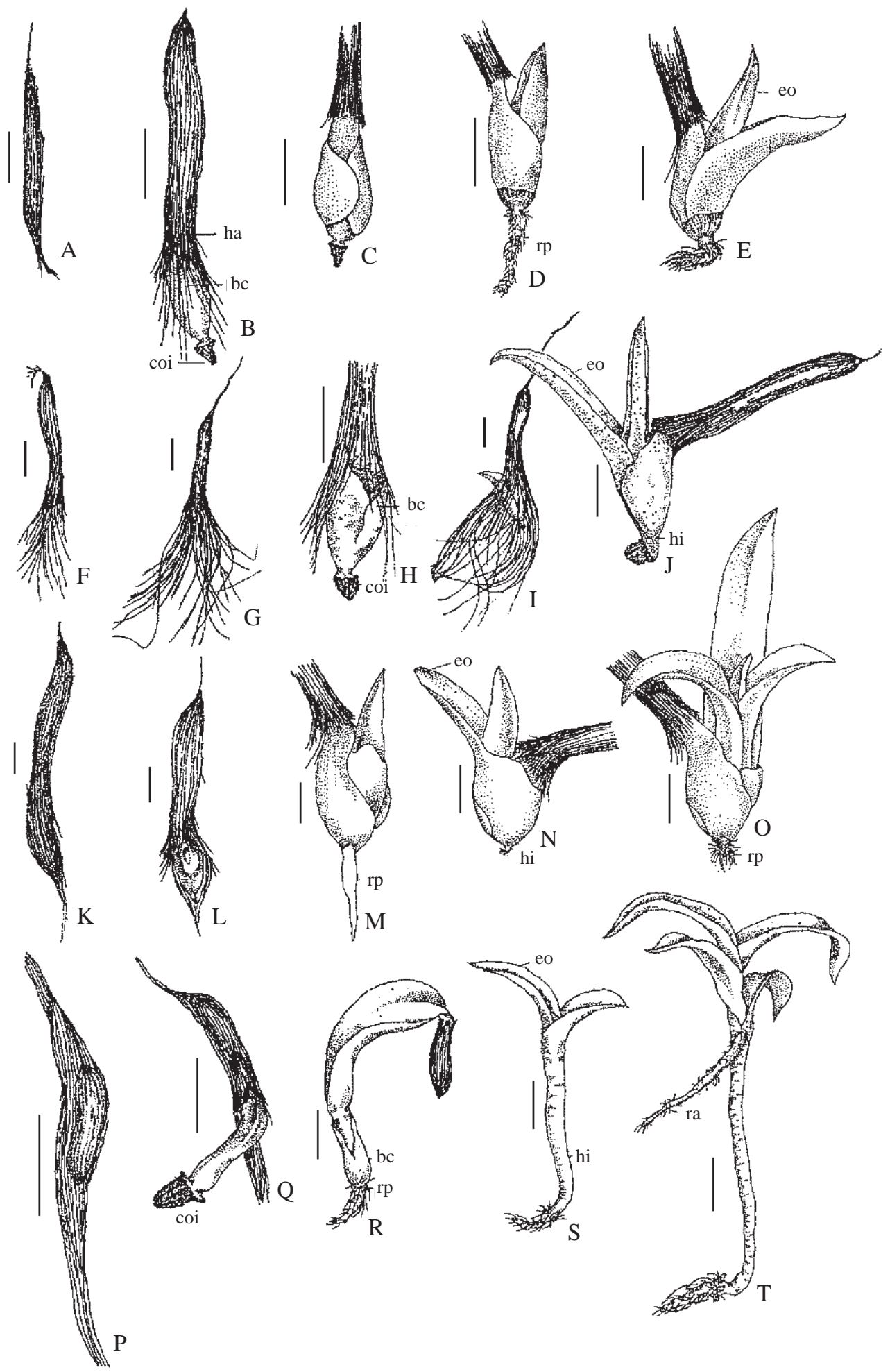

Figura 1. Morfologia da semente e do desenvolvimento pós-seminal de espécies de Bromeliaceae. A-E. Vriesea heterostachys. F-J. V. penduliflora. K-O. Alcantarea imperialis. P-T. Pitcairnia flammea. (bc = bainha cotiledonar; coi = coifa; eo = eófilo; ha $=$ haustório; hi = hipocótilo; ra = raiz adventícia; $r p=$ raiz primária). Escala $=1 \mathrm{~mm}$.

Figure 1. Seed and post-seminal development morphology of Bromeliaceae species. A-E. Vriesea heterostachys. F-J. V. penduliflora. K-O. Alcantarea imperialis. P-T. Pitcairnia flammea. $(\mathrm{bc}=$ cotyledonary sheath; $\mathrm{coi}=$ cap; eo = eophyll; ha = haustorium; hi = hypocotyl; ra = adventitious root; $\mathrm{rp}=$ primary root). $\mathrm{Bar}=1 \mathrm{~mm}$. 
Fatores abióticos no controle da germinação - As sementes das quatro espécies apresentaram alta germinabilidade $(>80 \%)$ sob fotoperíodo de oito horas. As sementes de A. imperialis, $V$. heterostachys e $V$. penduliflora, foram capazes de germinar em ampla faixa de temperaturas, entre $15^{\circ} \mathrm{C}$ e $35^{\circ} \mathrm{C}$, exceto $V$. penduliflora, também capaz de germinar em $10^{\circ} \mathrm{C}$ (figura 2). Para A. imperialis a temperatura de $30^{\circ} \mathrm{C}$ não diferiu estatisticamente $(P<0,05)$ de $20^{\circ} \mathrm{C}$ e $25^{\circ} \mathrm{C}$ em relação à porcentagem de germinação, mas foi significativamente superior em relação às demais temperaturas para a velocidade de germinação. Em $V$. heterostachys, $20^{\circ} \mathrm{C}$ foi a temperatura que produziu a maior porcentagem de germinação, não diferindo estatisticamente de $25^{\circ} \mathrm{C}, 30^{\circ} \mathrm{C}$ e $35^{\circ} \mathrm{C}$. A mais alta velocidade de germinação foi obtida na temperatura de $30{ }^{\circ} \mathrm{C}$, sendo significativamente distinta das demais temperaturas, exceto de $20^{\circ} \mathrm{C}$. Para $V$. penduliflora, as temperaturas entre $15^{\circ} \mathrm{C}$ e $35^{\circ} \mathrm{C}$ não diferiram significativamente para a porcentagem de germinação. Em contrapartida, a temperatura de $25^{\circ} \mathrm{C}$ foi a que apresentou a maior velocidade de germinação, não diferindo estatisticamente de $20^{\circ} \mathrm{C}$ e $30^{\circ} \mathrm{C}$. Em $P$. flammea, as temperaturas de $20^{\circ} \mathrm{C}$ e $25^{\circ} \mathrm{C}$ apresentaram a maior porcentagem e velocidade de germinação, estatisticamente superior as demais temperaturas, respectivamente. Não foi observada germinação na temperatura de $40{ }^{\circ} \mathrm{C}$ para as quatro espécies estudadas (figura 2). A alternância de temperaturas promoveu a germinação de forma semelhante às melhores temperaturas constantes $(P>0,05)$, independente da espécie estudada (resultados não apresentados).
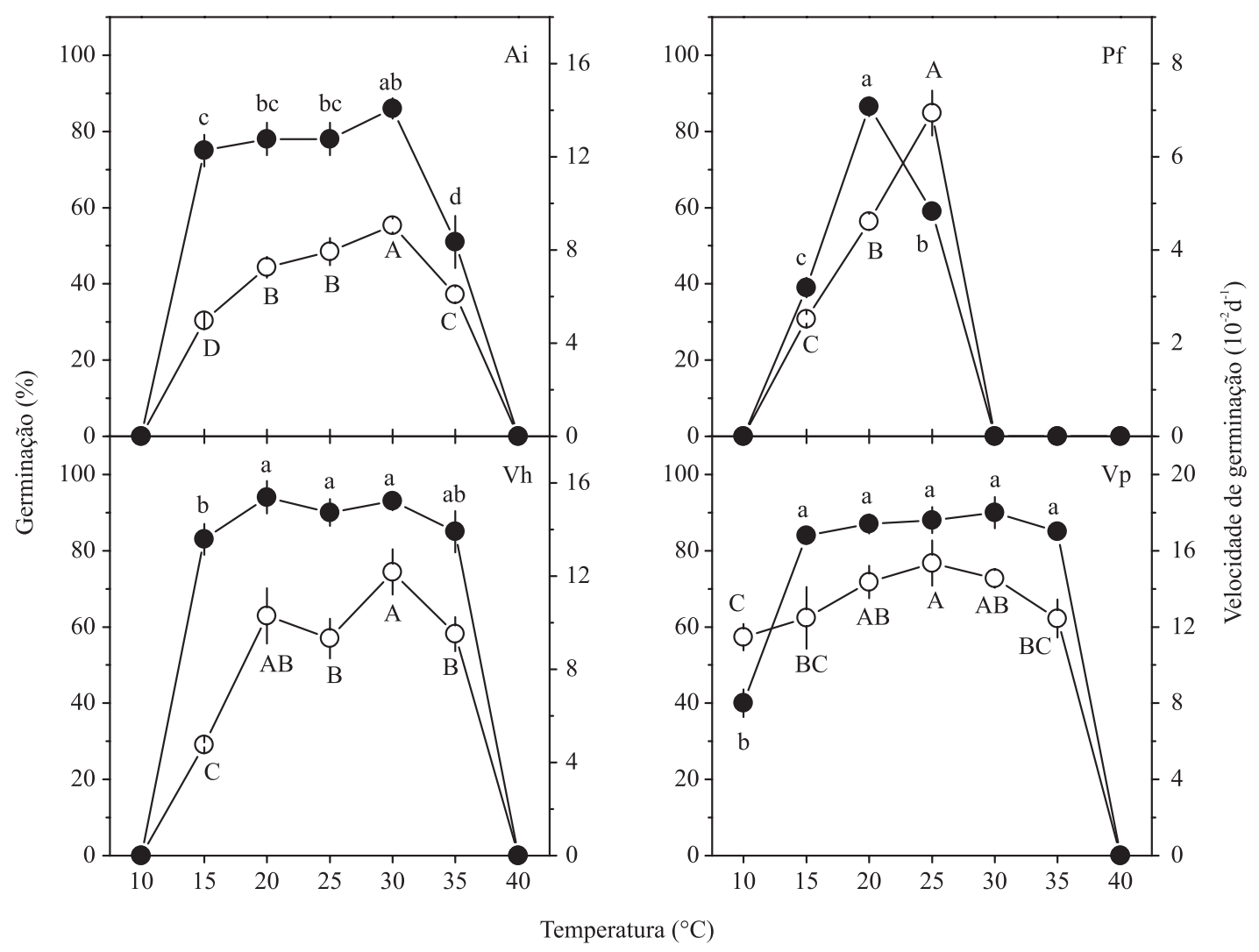

Figura 2. Porcentagem ( $\bullet$ ) e velocidade de germinação ( $\mathrm{O}$ ) de sementes de Alcantarea imperialis (Ai), Pitcairnia flammea (Pf), Vriesea heterostachys (Vh) e V. penduliflora (Vp) submetidas a diferentes temperaturas constantes, sob fotoperíodo de oito horas. Médias seguidas de mesma letra não diferem estatisticamente pelo teste de Tukey $(P<0,05)$. Barras verticais representam o desvio padrão, quando maior que os símbolos.

Figure 2. Final percentage $(\bullet)$ and rate $(\mathrm{O})$ of germination of Alcantarea imperialis (Ai), Pitcairnia flammea (Pf), Vriesea heterostachys $(\mathrm{Vh})$ e $V$. penduliflora $(\mathrm{Vp})$ seeds subjected to various constant temperatures, under eight hour photoperiod. Means followed by the same letter are not significantly different at the $P<0.05$ level (Tukey test). Vertical bars denote standard deviation when larger than symbols. 
Alcantarea imperialis e P. flammea apresentaram germinação nula sob ausência de luz. As sementes das duas outras espécies foram capazes de germinar no escuro, com valores médios de $18 \%$ para $V$. penduliflora e $52 \%$ para $V$. heterostachys. Todas as espécies estudadas apresentaram os mais altos valores de germinação quando submetidas à maior razão de $\mathrm{V}: \mathrm{VE}(1,2)$, sendo significativamente superiores (teste $t ; P<0,05$ ) ao tratamento de escuro contínuo (figura 3).

A função sigmoidal promoveu ajustes adequados para os dados observados, com coeficientes de regressão $\left(R^{2}\right)$ variando entre 0,93 e 0,98 (tabela 2). Independentemente da espécie, a redução dos valores de V:VE resultou no decréscimo da germinação. Entretanto, as quatro espécies apresentaram valores distintos de V:VE correspondente a $50 \%$ da máxima germinação ( $\mathrm{V}: \mathrm{VE}_{50 \% \text { Gmáx }}$ ), sendo muito mais altos para $A$. imperialis e $P$. flammea $(0,085$ e 0,122 , respectivamente) que para $V$. heterostachys e $V$. penduliflora $(0,013$ e 0,026, respectivamente).

Independente da espécie, tanto a porcentagem como a velocidade de germinação foram reduzidas com o decréscimo do $\Psi_{\mathrm{w}}$. Decréscimos significativos na porcentagem de germinação foram observados para $A$. imperialis, $P$. flammea, $V$. penduliflora e $V$. heterostachys a partir dos $\Psi_{\mathrm{w}}$ de -0,75 MPa, -0,25 MPa, -0,50 MPa e -0,50 MPa, respectivamente. A germinação nula também foi distinta entre as espécies, correspondendo aos $\Psi_{\mathrm{w}}$ de -1,25 MPa, -1,50 MPa, -1,25 MPa e -1,0 MPa para $A$. imperialis, $P$. flammea, V. penduliflora e V. heterostachys, respectivamente. Considerando a velocidade de germinação, reduções significativas em seus valores foram encontradas no $\Psi_{\mathrm{w}}$ de $-0,25 \mathrm{MPa}$, em todas as espécies estudadas (figura 4).

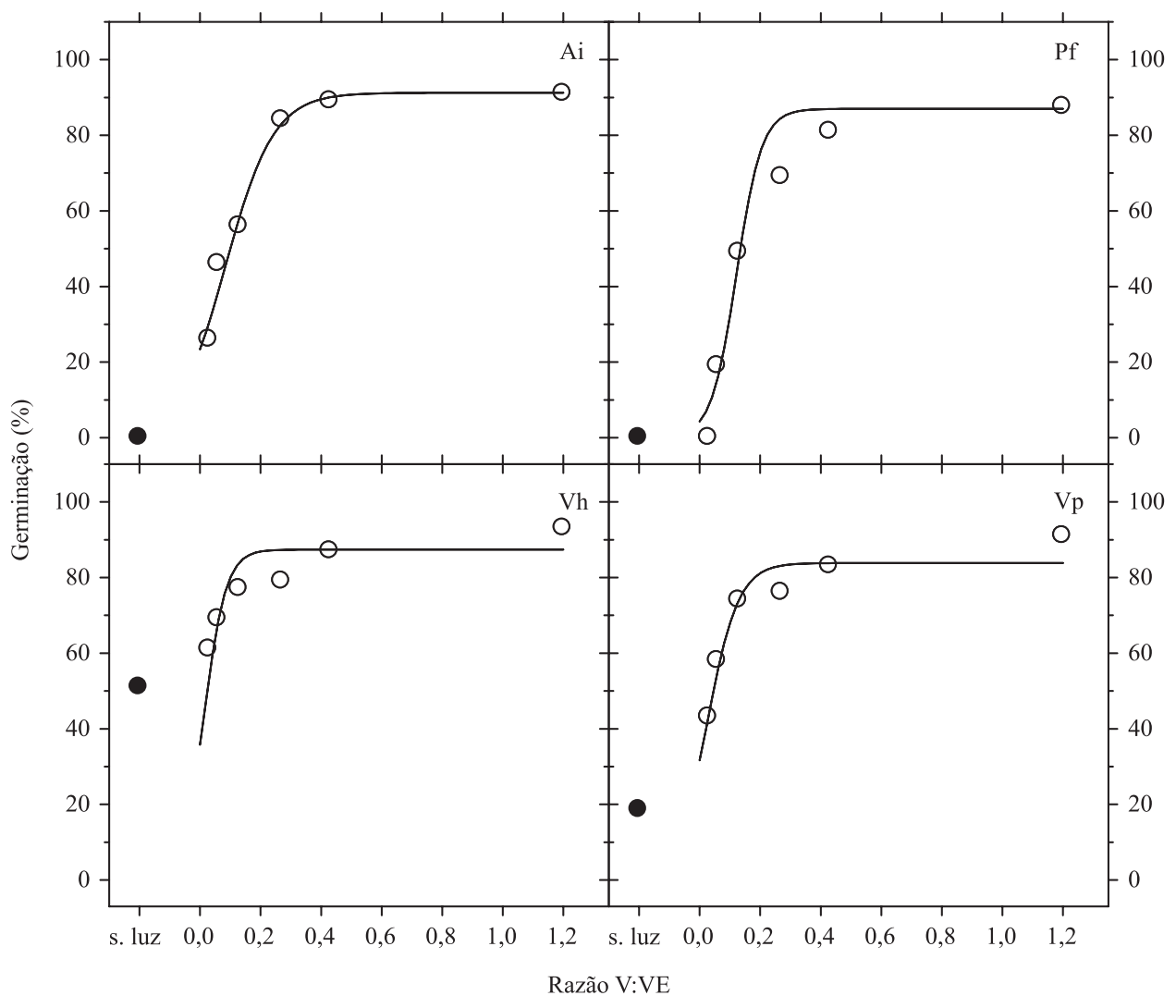

Figura 3. Germinação de sementes de Alcantarea imperialis (Ai), Pitcairnia flammea (Pf), Vriesea heterostachys (Vh) e V. penduliflora (Vp) submetidas a diferentes razões entre os comprimentos de onda vermelho e vermelho extremo (V:VE). Valores médios foram ajustados através de regressão sigmoidal logística (linha). Símbolos escuros para cada espécie representam a germinação no escuro (s. luz). Barras verticais representam o desvio padrão, quando maior que os símbolos.

Figure 3. The effect of R:FR ratio on mean final germination percentage of Alcantarea imperialis (Ai), Pitcairnia flammea (Pf), Vriesea heterostachys (Vh) e V. penduliflora (Vp) seeds. Mean values are fitted using sigmoidal regression (lines). For each species, closed symbols represent germination in the dark (s. luz). Vertical bars denote standard deviation when larger than symbols. 
Tabela 2. Coeficientes de regressão logística sigmoidal da relação V:VE e a germinação das quatro espécies de Bromeliaceae, onde $a$ descreve a máxima porcentagem de germinação, $b$ descreve a inclinação da resposta de germinação e $x_{0}$ estima o valor de V:VE equivalente a $50 \%$ da máxima germinação. Níveis de significância após os coeficientes de regressão $\left(R^{2}\right)$ apresentados a seguir: * $P=0,05 ; * * P=0,01$.

Table 2. Coefficients from sigmoidal regression of final germination percentage against R:FR for four Bromeliaceae seeds, where $a$ is a coefficient describing the maximum germination percentage, $b$ is a coefficient of the slope of the germination response, and $x_{0}$ is a coefficient estimating the R:FR at $50 \%$ of maximum germination. Degree of significance indicated as follows: * $P=0,05$; ** $P=0,01$.

\begin{tabular}{lcccc}
\hline Espécie & $a$ & $b$ & $x_{0}$ & $R^{2}$ \\
\hline Alcantarea imperialis & 91,24 & 12,54 & 0,095 & $0,98 * *$ \\
Pitcairnia flammea & 87,12 & 24,19 & 0,122 & $0,95 * *$ \\
Vriesea heterostachys & 87,40 & 27,45 & 0,013 & $0,93 *$ \\
Vriesea penduliflora & 83,85 & 19,47 & 0,026 & $0,95 *$ \\
\hline
\end{tabular}
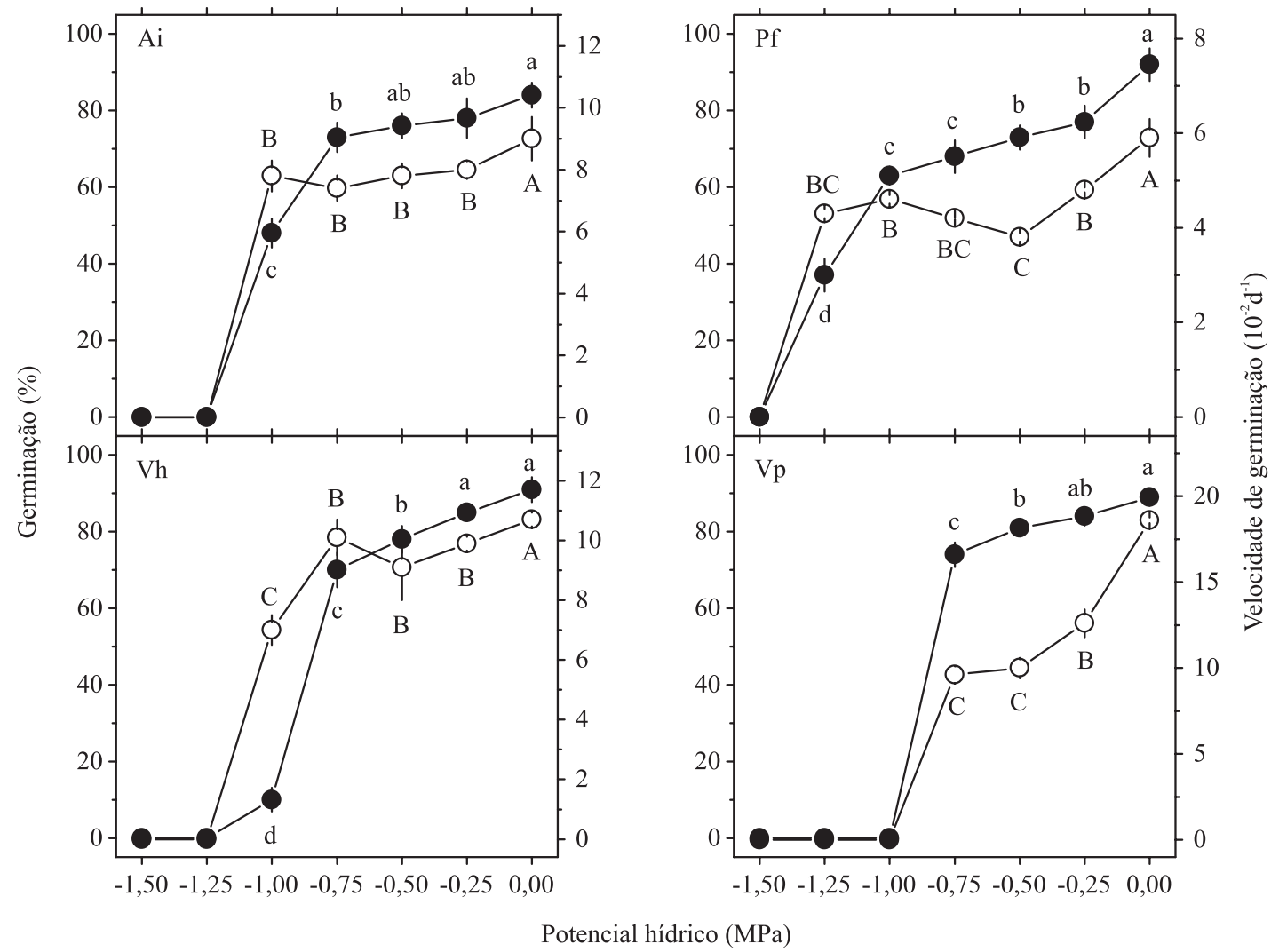

Figura 4. Porcentagem ( $\bullet$ ) e velocidade de germinação ( O ) de sementes de Alcantarea imperialis (Ai), Pitcairnia flammea (Pf), Vriesea heterostachys (Vh) e Vriesea penduliflora (Vp) submetidas a diferentes potenciais hídricos. Médias seguidas de mesma letra não diferem estatisticamente pelo teste de Tukey $(P<0,05)$. Barras verticais representam o desvio padrão, quando maior que os símbolos.

Figure 4. Final percentage $(\bullet)$ and rate $(\mathrm{O})$ of germination of Alcantarea imperialis (Ai), Pitcairnia flammea (Pf), Vriesea heterostachys (Vh) e Vriesea penduliflora $(\mathrm{Vp})$ seeds subjected to various water potentials. Means followed by the same letter are not significantly different at the $P<0.05$ level (Tukey test). Vertical bars denote standard deviation when larger than symbols. 


\section{Discussão}

Em relação ao desenvolvimento pós-seminal, as espécies $A$. imperialis, $V$. heterostachys e $V$. penduliflora foram caracterizadas pela germinação criptoepígea, embora não apresentem hipocótilo desenvolvido como P. flammea. Segundo Boyd (1932), as características que condicionam a germinação epígea em Bromeliaceae estão relacionadas à tendência ao epifitismo e à ausência ou rara presença de feixes vasculares na bainha cotiledonar. Para P. flammea, a germinação iniciou-se com o rompimento do tegumento e protrusão da raiz primária, enquanto que, para as espécies de Tillandsioideae, a germinação iniciou-se com a emergência da base do cotilédone, e posteriormente desenvolveu-se a raiz primária. Scatena et al. (2006) observaram em espécies de Tillandsia que a primeira estrutura que emergiu durante a germinação foi o cotilédone haustorial e não apresentaram raiz primária.

Para a maioria das bromélias rupícolas e terrestres, suas plântulas apresentam considerável volume de raízes espessas e funcionais (Paula \& Silva 2004). No entanto, A. imperialis, cuja morfologia radicular na fase inicial de germinação foi similar às das espécies epífitas, apresentou sistema radicular fasciculado e pouco desenvolvido. Isto sugere que a história evolutiva destas plantas, onde Vriesea e Alcantarea emergem como grupos intimamente relacionados (Barfuss et al. 2005) é mais importante que o ambiente que elas colonizam. É provável que os tricomas foliares tenham uma função fundamental para esta espécie, auxiliando na absorção de água e nutrientes durante o desenvolvimento subseqüente do sistema radicular, corroborando o padrão descrito na literatura para as espécies de Tillandsioideae (Benzing 2000, Scatena et al. 2006).

O tipo de plântula observado para as espécies de Bromeliaceae aqui estudadas foi criptocotiledonar/ epígea com cotilédone de reserva (CER), segundo a classificação de Garwood (1996). Apesar das espécies estudadas apresentarem formas de vida diferentes, com condições ambientais de crescimento distintas (luminosidade, temperatura e disponibilidade de água) e diferenças morfológicas que distinguem bem as duas subfamílias, todas possuem o mesmo tipo de plântula. De acordo com Garwood (1996), o tipo de plântula é uma característica conservativa, com forte inércia filogenética para gêneros e famílias, sugerindo a estabilidade desse caráter, mesmo em táxons distantes.

As quatro espécies produzem sementes muito leves, com massa individual das sementes variando entre 0,16 a 1,7 mg e síndrome anemocórica de dispersão, apresentando estruturas aladas do tipo alas membranáceas ou apêndices plumosos, presentes em uma ou nas duas extremidades da semente. De acordo com Benzing (2000) e Scatena et al. (2006), tais características facilitariam a dispersão das sementes por grandes distâncias e permitiriam que elas alcançassem e se estabelecessem em microambientes inóspitos. A eficiência deste tipo de dispersão, aliada à quantidade massiva de sementes pequenas e com baixa disponibilidade de reservas internas produzidas na maturação, poderia indicar a baixa probabilidade de sobrevivência destas espécies na fase inicial de germinação, como sugerido por Meyer \& Carlson (2001). Dessa forma, a detecção de microambientes favoráveis à germinação é fundamental para o estabelecimento dessas espécies, definindo a sua distribuição entre diferentes habitats (Grubb 1977).

As sementes das quatro espécies germinaram na faixa de temperaturas entre $15^{\circ} \mathrm{C}$ e $35^{\circ} \mathrm{C}$. Exceção foi observada em $P$. flammea, capaz de germinar apenas entre $15^{\circ} \mathrm{C}$ e $25^{\circ} \mathrm{C}$. Resultados semelhantes foram obtidos para sementes de P. flammea coletadas em São Paulo (Mercier \& Guerreiro Filho 1990). A faixa ideal para a germinação das bromélias estudadas foi obtida entre $20^{\circ} \mathrm{C}$ e $30^{\circ} \mathrm{C}$, corroborando com as informações de Mercier \& Guerreiro Filho (1990) para oito espécies de bromélias da Mata Atlântica, e a de Borghetti (2005) quanto à faixa ótima de germinação para as sementes de espécies tropicais. A ausência de germinação em temperaturas sub ou supra-ótimas pode ser resultado do processo de termodormência, considerado um mecanismo ecológico de grande importância na prevenção da germinação sob condições ambientais desfavoráveis (Baskin \& Baskin 2001).

De maneira semelhante a muitas espécies com sementes pequenas (Milberg et al. 2000), todas as espécies estudadas produziram alta germinabilidade apenas quando submetidas as maiores intensidades de luz e de $\mathrm{V}$ :VE. Entretanto, as quatro espécies apresentaram dois comportamentos distintos em relação aos tratamentos V:VE. As duas espécies rupícolas (A. imperialis e $P$. flammea) tiveram sua germinação fortemente inibida quando suas sementes foram submetidas aos baixos valores de V:VE, confirmando as condições de luminosidade encontradas em suas áreas de ocorrência, ou seja, locais com exposição direta à luz solar. O mecanismo de detecção de qualidade e quantidade de luz pelo fitocromo pode explicar a prevenção à germinação em condições encontradas sob luz difusa de florestas fechadas (Pons 2000). As sementes destas duas espécies podem ser classificadas como fotoblásticas positivas, pois não foram capazes de germinar no escuro mesmo 
quando submetidas à alternância de temperatura (20$30^{\circ} \mathrm{C}$ ). Tal comportamento pode ser ecologicamente importante, pois impede que as sementes germinem quando soterradas no solo ou em fendas de rochas com nenhuma luz, esgotando suas reservas antes que suas plântulas alcancem espectros fotossinteticamente ativos de luz nas camadas mais superficiais, situação comum para as duas espécies rupestres.

As sementes das espécies epífitas encontradas na área florestal, V. heterostachys e V. penduliflora, coletadas no sub-bosque de floresta ombrófila e no dossel e borda da mata nebular, respectivamente, foram capazes de germinar no escuro (G\% > 20\%), sendo consideradas desta forma como fotoblásticas neutras ou fotoblásticas relativas (sensu Ferreira et al. 2001). Além disso, mesmo os baixos valores de V:VE utilizados neste estudo ( $\mathrm{V}: \mathrm{VE} \cong 0,03$ ), cerca de seis vezes mais baixos que aqueles encontrados no sub-bosque de florestas tropicais (Pearson et al. 2002), promoveram a germinação com taxas acima de $40 \%$. Neste caso, as sementes seriam capazes de germinar após a exposição à luz filtrada pela serapilheira e/ou solo, quando enterradas em pequenas profundidades ou pelas folhas das copas das árvores, quando aderidas aos troncos e galhos após a dispersão, permitindo a sua germinação sob condições sombreadas no interior da floresta, onde a intensidade de luz e os valores de V:VE são menores, conforme sugerido por Capers \& Chazdon (2004). Para estas espécies, outros fatores podem estar selecionando locais favoráveis à germinação, tais como temperatura e disponibilidade hídrica.

Em relação à disponibilidade hídrica do substrato, as sementes das quatro espécies apresentaram decréscimo da porcentagem e da velocidade de germinação, com a redução do $\Psi_{\text {w }}$ Entretanto, foi possível observar que as duas espécies rupícolas (A. imperialis e $P$. flammea) foram capazes de germinar sob $\Psi_{\mathrm{w}}$ mais negativo que as espécies epífitas ( $V$. heterostachys e $V$. penduliflora). $\mathrm{O}$ ambiente onde $A$. imperialis e $P$. flammea foram encontradas caracteriza-se pela alta irradiação solar, baixa disponibilidade de água no solo raso ou ausente, além dos ventos fortes, sujeito a situações de déficit hídrico muito mais intenso que os ambientes epifíticos das outras duas espécies. Alcantarea imperialis e P. flammea apresentaram germinação moderada (G\% > 40\%) sob $\Psi_{\mathrm{w}}$ de -1,25 MPa e de -1,0 MPa, respectivamente. Resultados semelhantes foram obtidos para sementes de Mammillaria gaumeri (Britton \& Rose) Orcutt, Agriophyllum squarrosum (L.) Moq. e Indigofera astragalina DC., oriundas de regiões extremamente secas, apresentando germinação mediana nos $\Psi_{\mathrm{w}}$ de -1,0 MPa, -1,01 MPa, -1,5 MPa e
-1,4 MPa, respectivamente (Cervera et al. 2006, Sy et al. 2001, Tobe et al. 2005). No entanto, V. heterostachys e $V$. penduliflora germinaram em taxas semelhantes até -0,75 MPa. Os valores encontrados para as espécies epífitas foram muito próximos aos encontrados para sementes de espécies florestais de áreas úmidas (Baskin \& Baskin 2001). De acordo com Evans \& Etherington (1990), espécies que crescem em ambientes secos produzem sementes capazes de germinar sob $\Psi_{\mathrm{w}}$ mais negativos que espécies de ambientes úmidos. Quando comparadas a espécies adaptadas a ambientes secos (Baskin \& Baskin 2001), o $\Psi_{\mathrm{w}}$ no qual a germinação das bromélias rupestres foi inibida mostrou-se superior. Além do aspecto fisiológico, as diferenças morfológicas na fase pós-seminal, principalmente em relação ao sistema radicular mais desenvolvido, possivelmente, permitirá que $P$. flammea absorva com mais eficiência água para o estabelecimento das plântulas.

Quando submetidas a condições microambientais favoráveis, as sementes das quatro espécies não apresentaram dormência primária, resultando em uma alta e rápida germinação. Segundo Garwood (1996), tal característica sugere que o banco de sementes não faz parte das estratégias de regeneração destas espécies. Uma possível estratégia utilizada em Bromeliaceae, principalmente quando as condições climáticas não se apresentam favoráveis durante o processo germinativo das sementes, é a emissão de rametes por propagação assexuada. Dessa forma, tais espécies são capazes de colonizar ambientes hostis e conseguem se perpetuar (Zaluar \& Scarano 2000).

As exigências específicas para a germinação de muitas espécies estão associadas à sua forma de vida, à época de frutificação e ao ambiente onde a planta será estabelecida (Flores \& Briones 2001). Desta forma, é possível concluir que as duas bromélias epífitas ( $V$. heterostachys e V.penduliflora) apresentam como principal fator limitante à germinação os níveis de hidratação do substrato, pois germinam em ampla faixa de temperaturas, independente da condição de luz (fotoblásticas neutras), mas não germinam abaixo de $\Psi_{\mathrm{w}}=-1 \mathrm{MPa}$, como as duas espécies rupícolas. Estes resultados corroboram a análise de Lüttge (2008), que considera a disponibilidade hídrica como a principal restrição ao hábito epifítico. Assim, as duas espécies estariam mais aptas a germinar em locais sombreados, e consequentemente mais úmidos do interior de florestas. Em contrapartida, A. imperialis e $P$. flammea apresentam características germinativas que favorecem o seu estabelecimento em ambientes rupestres, em locais submetidos intensidades elevadas de radiação solar e estresse hídrico de moderado a intenso. 
Possivelmente, a faixa restrita de temperaturas encontrada para a germinação de $P$. flammea está relacionada à época de maturação de seus frutos e sementes na região de Ibitipoca-MG, concentrada entre o fim do inverno e princípio da primavera, quando as temperaturas ainda não são elevadas.

Agradecimentos - Ao CNPq (auxílio à pesquisa, bolsa de doutorado de A.R.P., bolsa de produtividade de R.C.F.) e Capes pelo suporte financeiro e ao Programa de PósGraduação em Botânica do Jardim Botânico do Rio de Janeiro. Este trabalho foi parcialmente financiado pelo projeto "Variabilidade genética, propagação in vitro, germinação e conservação de espécies ameaçadas de Bromeliaceae”, Fundação Biodiversitas.

\section{Referências bibliográficas}

BARFUSS, M.H.J., SAMUEL, R., TILL, W. \& STUESSY, T.F. 2005. Phylogenetic relationships in subfamily Tillandsioideae (Bromeliaceae) based on DNA sequence data from seven plastid regions. American Journal of Botany 92:337-351.

BASKIN, C.C. \& BASKIN, J.M. 2001. Seeds: ecology, biogeography, and evolution of dormancy and germination. Academic Press, San Diego.

BENZING, D.H. 2000. Bromeliaceae: profile of an adaptive radiation. Cambridge University Press, Cambridge.

BORGHETTI, F. 2005. Temperaturas extremas e a germinação de sementes. In Estresses ambientais, danos e benefícios em plantas. (R.J.M.C. Nogueira, E.L. Araújo, L.G. Willadino \& U.M.T. Cavalcante, eds.). MXM Gráfica e Editora, Recife, p.207-218.

BOYD, L. 1932. Monocotylous seedlings. Morphological studies in the post-seminal development of the embryo. Transactions and Proceedings of the Botanical Society of Edinburgh 31:5-224.

BRASIL. 1992. Regras para análise de sementes. Ministério da Agricultura e Reforma Agrária. LAVARV/ SNAD/ MA, Brasília.

BURKE, A. 2002. Island - matrix relationships in Nama Karoo inselberg landscapes Part II. Are some inselbergs better sources than others? Plant Ecology 158:41-48.

CAPERS, R.S. \& CHAZDON, R.L. 2004. Rapid assessment of understory light availability in a wet tropical forest. Agricultural and Forest Meteorology 123:177-185.

CERVANTES, S.E., GRAHAM, E.A. \& ANDRADE, J.L. 2005. Light microhabitat, growth and photosynthesis of an epiphytic bromeliad in a tropical dry forest. Plant Ecology 179:107-118.

CERVERA, J.C., ANDRADE, J.L., SIMÁ, J.L. \& GRAHAMT, E.A. 2006. Microhabitats, germination, and establishment for Mammillaria gaumeri (Cactaceae), a rare species from Yucatan. International Journal Plant Sciences 16:311-319.
COAN, A.I., SCATENA, V.L. \& GIULIETTI, A.M. 2002. Anatomia de algumas espécies aquáticas de Eriocaulaceae brasileiras. Acta Botanica Brasilica 16:371-384.

DIAS, H.C.T., FERNANDES-FILHO, E.I., SCHAEFER, C. E.G.R., FONTES, L.E.F. \& VENTORIM, L.B. 2002. Geoambientes do Parque Estadual do Ibitipoca, município de Lima Duarte-MG. Revista Árvore 26:777-786.

EVANS, C.E. \& ETHERINGTON, J.R. 1990. The effect of soil water potential on seed germination of some British plants. New Phytologist 115:539-548.

FENNER, M. \& THOMPSON, K. 2005. The ecology of seeds. Cambridge University Press, Cambridge.

FERREIRA, A.G., CASSOL, B., ROSA, S.G.T., SILVEIRA, T.S., STIVAL, A.L. \& SILVA, A.A. 2001. Germinação de sementes de Asteraceae nativas no Rio Grande do Sul, Brasil. Acta Botanica Brasilica 15:231-242.

FLORES, J. \& BRIONES, O. 2001. Plant life-form and germination in a Mexican inter-tropical desert: effects of soil water potential and temperature. Journal of Arid Environments 47:485-497.

GARWOOD, N.C. 1996. Functional morphology of tropical tree seedlings. In The ecology of tropical forest tree seedlings (M.D. Swaine ed.). The Parthenon Publishing Group, Paris.

GRAHAM, E.A. \& ANDRADE, J.L. 2004. Drought tolerance associated with vertical stratification of two co-occurring epiphytic bromeliads in a tropical dry forest. American Journal of Botany 91:699-706.

GRIME, J.P. 2001. Plant strategies, vegetation processes, and ecosystem properties. $2^{\text {nd }}$ ed. Wiley, Chichester.

GRUBB, P.J. 1977. The maintenance of species richness in plant communities: the importance of the regeneration niche. Biological Review 52:107-145.

HARPER, J.L. 1977. Population biology of plants. Academic Press, London.

LEME, E.M.C. 1997. Bromélias da Mata Atlântica: Canistrum. Salamandra. Rio de Janeiro.

LUTHER, H.E. 2008. An alphabetical list of bromeliad binomials. $11^{\text {th }}$ ed. The Bromeliad Society International, Sarasota.

LÜTTGE, U. 2008. Physiological ecology of tropical plants. Springer-Verlag, Berlin.

MENINI NETO, L., ALVES, R.V., BARROS, F. \& FORZZA, R.C. 2007. Orchidaceae do Parque Estadual de Ibitipoca, Minas Gerais, Brasil. Acta Botanica Brasilica 21:687696.

MERCIER, H. \& GUERREIRO FILHO, O. 1990. Propagação sexuada de algumas bromélias nativas da Mata Atlântica: efeito da luz e da temperatura na germinação. Hoehnea 17:19-26.

MEYER, S.E. \& CARLSON, S.L. 2001. Achene mass variation in Ericameria nauseosus (Asteraceae) in relation to dispersal ability and seedling fitness. Functional Ecology 15:274-281. 
MICHEL, B.E. \& KAUFMANN, M.R. 1973. The osmotic potential of polyethylene glycol 6000. Plant Physiology 51:914-916.

MILBERG, P., ANDERSSON, L. \& THOMPSON, K. 2000. Large-seeded species are less dependent on light for germination than small-seeded ones. Seed Science Research 10:99-104.

MONTEIRO, R.F. \& FORZZA, R.C. 2008. A família Bromeliaceae no Parque Estadual do Ibitipoca, Minas Gerais, Brasil. Boletim de Botânica da Universidade de São Paulo 26:7-33.

PAULA, C.C. \& SILVA, H.M.P. 2004. Cultivo prático de bromélias. UFV, Viçosa.

PEARSON, T.R.H., BURSLEM, D.F.R.P., MULLINS, C.E. \& DALLING, J.W. 2002. Germination ecology of neotropical pioneers: interacting effects of environmental conditions and seed size. Ecology 83: 2798-2807.

PEARSON, T.R.H., BURSLEM, D.F.R.P., MULLINS, C.E. \& DALLING, J.W. 2003. Functional significance of photoblastic germination in neotropical pioneer tress: a seed's eye view. Functional Ecology 17:394-402.

PEREIRA, T.S. 1988. Bromelioideae (Bromeliaceae): morfologia do desenvolvimento pós-seminal de algumas espécies. Arquivo do Jardim Botânico do Rio de Janeiro 29:115-154.

PONS, T.L. 2000. Seed responses to light. In Seeds: the ecology of regeneration in plant communities. (M. Fenner, ed.). CABI Publishing, Wallingford, p.237-260.

RIBEIRO, K.T., MEDINA, B.M.O. \& SCARANO, F.R. 2007. Species composition and biogeographic relations of the rock outcrop flora on the high plateau of Itatiaia, SE-Brazil. Revista Brasileira de Botânica 30:623-639.
RODELA, L.G. \& TARIFA, J.R. 2002. O clima da Serra de Ibitipoca, sudeste de Minas Gerais. GEOUSP - Espaço e Tempo 11:101-113.

SANTANA, D.G. \& RANAL, M.A. 2004. Análise da germinação - um enfoque estatístico. Universidade de Brasília, Brasília.

SCATENA, V.L., SEGECIN, S. \& COAN, A.I. 2006. Seed morphology and post-seminal development of Tillandsia L. (Bromeliaceae) from the "Campos Gerais”, Paraná, Southern Brazil. Brazilian Archives of Biology and Technology 49:945-951.

STATSOFT, INC. 2001. Statistica (data analysis software system), version 6. Tulsa, OK. <http://www.statsoft. com> (acesso em 22 ago. 2007).

SY, A., GROUZIS, M. \& DANTHU, P. 2001. Seed germination of seven Sahelian legume species. Journal of Arid Environments 49:875-882.

TILLICH, H.J. 2007. Seedling diversity and the homologies of seedling organs in the order Poales (Monocotyledons). Annals of Botany 100:1413-1429.

TOBE, K., ZHANG, L. \& OMASA, K. 2005. Seed germination and seedling emergence of three annuals growing on desert sand dunes in China. Annals of Botany 95:649-659.

VAN ASSCHE, J., VAN NERUM, D. \& DARIUS, P. 2002. The comparative germination ecology of nine Rumex species. Plant Ecology 159:131-142.

ZALUAR, H.L.T. \& SCARANO, F.R. 2000. Facilitação em restingas de moitas: um século de buscas por espécies focais. In Ecologia de restingas e lagoas costeiras. (F.A. Esteves \& L.D. Lacerda, eds.). Nupem-UFRJ, Rio de Janeiro, p.3-23.

ZAR, J.H. 1999. Biostatistical analysis. $4^{\text {th }}$ ed. Prentice-Hall, New Jersey. 